\title{
OPEN Low-flux scanning electron diffraction reveals substructures inside the ordered membrane domain
}

\begin{abstract}
Masanao Kinoshita ${ }^{\bowtie}$, Shimpei Yamaguchi \& Nobuaki Matsumori
Ordered/disordered phase separation occurring in bio-membranes has piqued researchers' interest because these ordered domains, called lipid rafts, regulate important biological functions. The structure of the ordered domain has been examined with artificial membranes, which undergo macroscopic ordered/disordered phase separation. However, owing to technical difficulties, the local structure inside ordered domains remains unknown. In this study, we employed electron diffraction to examine the packing structure of the lipid carbon chains in the ordered domain. First, we prepared dehydrated monolayer samples using a rapid-freezing and sublimation protocol, which attenuates the shrinkage of the chain-packing lattice in the dehydration process. Then, we optimised the electron flux to minimise beam damage to the monolayer sample. Finally, we developed low-flux scanning electron diffraction and assessed the chain packing structure inside the ordered domain formed in a distearoylphosphatidylcholine/dioleoylphosphatidylcholine binary monolayer. Consequently, we discovered that the ordered domain contains multiple subdomains with different crystallographic axes. Moreover, the size of the subdomain is larger in the domain centre than that near the phase boundary. To our knowledge, this is the first study to reveal the chain packing structures inside an ordered domain.
\end{abstract}

Cell membranes, which consist of a large variety of phospholipids, show lateral heterogeneity in terms of lipid distribution. Such heterogeneous distribution leads to ordered/disordered phase separation. Since the late twentieth century, researchers have been interested in the ordered membrane domains due to their potential involvement in important biological functions ${ }^{1}$. For instance, it is widely believed that some membrane proteins are transiently entrapped in ordered domains, facilitating transmembrane signalling. Despite their hypothesised importance in membrane biology, detailed information on ordered domains is limited, owing to their low spatiotemporal stability $^{2-4}$; the putative size of the domains in quiescent cells is less than $200 \mathrm{~nm}$ and their lifetime is less than a few hundred milliseconds.

So far, the physicochemical properties and structure of the ordered domains have been examined with artificial mono- and bi-layer membranes. For example, binary mixtures of saturated phospholipids or sphingomyelins (SMs) with unsaturated phospholipids undergo phase segregation between the ordered and disordered membrane domains ${ }^{5,6}$. In the case of bilayer systems, the addition of cholesterol (chol) to the binary mixtures forms another type of ordered domain called liquid ordered (Lo) phase $\mathrm{e}^{7-11}$. Because these ordered domains are more stable and significantly larger than those formed in bio-membranes, artificial membranes are useful to obtain basic information on ordered domains such as geometry ${ }^{12,13}$, lipid composition ${ }^{7,14,15}$, and thermal stability ${ }^{16}$. Recently, some studies have indicated that ordered domains are not homogeneous but consist of multiple subdomains. For example, a high-speed single-particle tracking experiment demonstrated that a gold nanoparticle labelled dipalmitoyl-phosphatidylethanolamine (GNP-DPPE) does not have simple Brownian motion in ordered domains, which are formed in dipalmitoylphosphatidylcholine (DPPC)/diphytanoylphosphatidylcholine/cholesterol (chol) ternary supported bilayers ${ }^{17}$. They speculated that GNP-DPPE is entrapped in the subdomains, hampering its normal diffusion in the ordered domain. Moreover, fluorescent lifetime measurements ${ }^{18}$ and Raman spectroscopy ${ }^{19}$ revealed that SMs forms small aggregations in SM/dioleoylphosphatidylcholine (DOPC) binary bilayers, while fluorescent microscopy identified the macroscopic ordered/disordered phase separation in these mixtures ${ }^{5}$. These facts indicate that aggregations of SMs exist in macroscopic ordered domains ${ }^{18}$. Although 


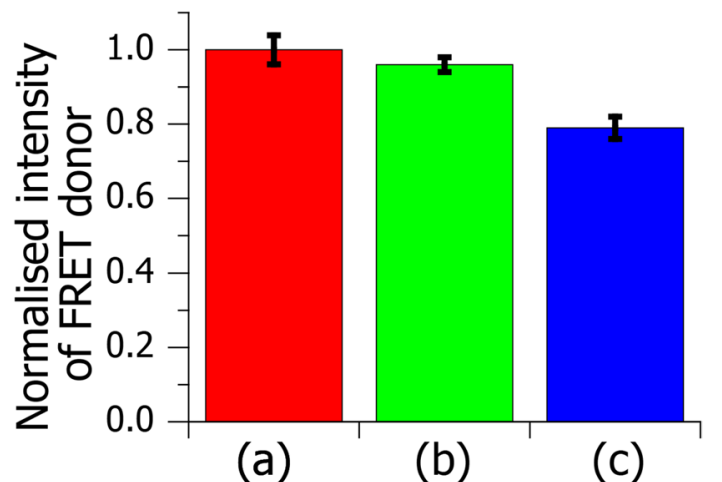

Figure 1. FRET-quenching intensity of the donor in wet and dehydrated DOPC monolayers. The samples contain $0.2 \mathrm{~mol} \%$ Bodipy-PC and $0.4 \mathrm{~mol} \%$ Texas Red labelled DPPE (Texas Red-DPPE) as FRET donor and acceptor, respectively. Bars show the donor intensity $I_{\text {donor }}$ of (a) a wet sample, (b) a sample dehydrated by the RFS method and (c) a sample dehydrated at the atmospheric pressure and room temperature. For easy comparison, the $I_{\mathrm{donor}}$-values of the dehydrated samples were normalised to that of the wet sample. Error bars indicate the standard errors.

there are several lines of evidence for the existence of subdomains ${ }^{20}$, the techniques used could not address lipid packing structures inside the ordered membrane domain.

Wide angle X-ray diffraction (WAXD) has been used for the investigation of lipid packing structures in artificial membranes ${ }^{21-24}$ because the WAXD shows diffraction peaks corresponding to lattice spacings of the lipid carbon chains. However, the diameter of the X-rays frequently used for those experiments is more than $100 \mu \mathrm{m}$, which is much larger than the size of the ordered domain. Consequently, a WAXD pattern contains integrated information on a number of ordered and disordered domains residing within the beam size. In addition, owing to the limitation of the scattering efficiency of the X-rays, the WAXD peaks from a single membrane domain are too weak to obtain reliable results. Therefore, another method is needed to examine the local structures inside a single ordered domain.

Instead of WAXD, electron diffraction (ED) is a promising method for local-structure analysis due to the following reasons: First, because ED is performed with an electron microscope, we can direct the electron beam to a micro-size target under visual observation. Second, using a selector aperture, we can select the ED patterns only from the desired regions, whose size is less than $1 \mu \mathrm{m}$ in diameter. Additionally, due to its significantly greater diffraction potency than X-rays, ED likely provides strong diffraction peaks even from a single membrane domain. While ED experiments were sometimes used for structural analysis of lipid membranes from the 1970s to the $1990 \mathrm{~s}^{25-32}$, the techniques progression has been hampered by some technical difficulties. For example, ED requires a dry and fixed sample because it is performed under a high vacuum. In addition, compared with $\mathrm{X}$-rays, the electron beam causes significant damage to the lipid membranes ${ }^{33,34}$.

In the present study, we prepare dehydrated samples employing a rapid-freezing and sublimation (RFS) protocol, hampering the shrinkage of the chain-packing lattice in the dehydration process. In addition, we optimised electron flux to minimise beam damage to the lipid membranes. Finally, we revealed the local structures inside a single ordered domain by newly developed low-flux scanning electron diffraction (LFSED).

\section{Results}

Influence of the rapid-freezing and sublimation (RFS) on the chain packing in lipid monolayer. It has previously been reported that dehydration of lipid membranes causes shrinkage of the chain packing lattice effectively in the disordered membranes ${ }^{25}$. To overcome this problem, we employed the RFS method to prepare dehydrated samples (see "Materials and methods" for details). Because lipid diffusion is fixed through the RFS protocol, it should create dehydrated samples without the shrinkage of the chain-packing lattice.

This could be confirmed by fluorescent energy transfer (FRET) measurements. Since FRET sensitively depends on the distance between the FRET donor and acceptor in membranes, shrinkage of the chain-packing lattice causes effective FRET-quenching of the donor fluorescence. Figure 1 shows normalised donor intensity $I_{\text {donor }}$ in DOPC supported monolayers, which form a homogeneous disordered membrane on the air-water interface ${ }^{35}$. The dehydration of the sample at atmospheric pressure and room temperature caused effective FRET-quenching of the donor intensity (compare Fig. 1a and c), indicating that the packing shrinkage occurs in the dehydration

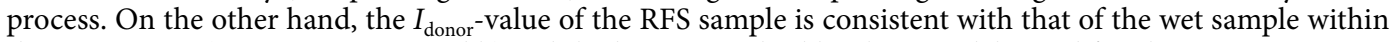
the error range (compare Fig. 1a and b). While the RFS method has been widely used for electron microscopy and mass-based imaging ${ }^{36-38}$, we demonstrated for the first time that it does not cause significant shrinkage of the chain packing lattice in the disordered monolayer.

Kinetics of structural decay caused by electron beam irradiation. We optimised the flux of the electron beam to minimise beam damage to the sample. Here, we used a distearoylphosphatidylcholine (DSPC) 


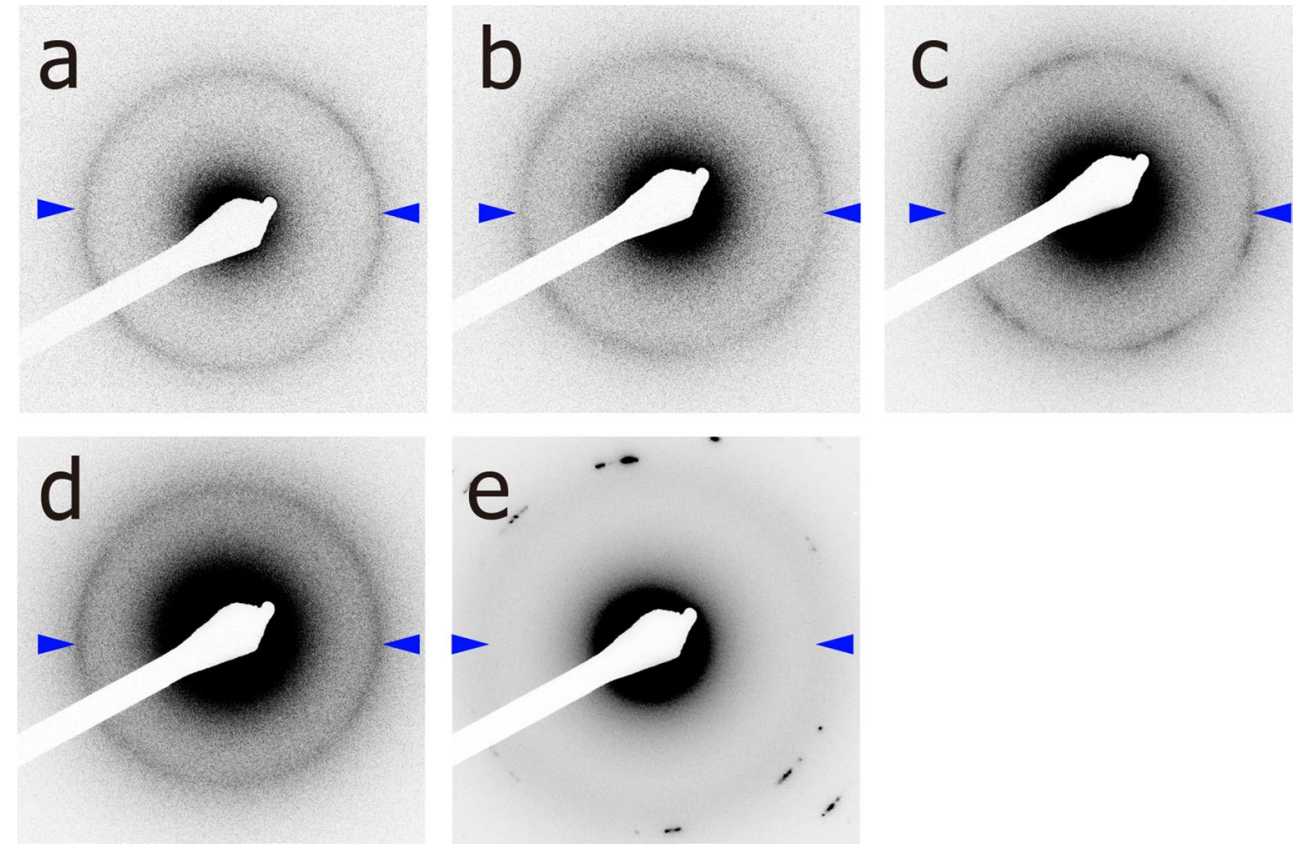

Figure 2. ED patterns from the DSPC monolayer at different electron fluxes; (a) $0.9 \mathrm{e} / \mathrm{nm}^{2} \cdot \mathrm{s},(\mathbf{b}) 2.3 \mathrm{e} / \mathrm{nm}^{2} \cdot \mathrm{s},(\mathbf{c})$ $4.7 \mathrm{e} / \mathrm{nm}^{2} \cdot \mathrm{s}$, (d) $15.9 \mathrm{e} / \mathrm{nm}^{2} \cdot \mathrm{s}$, and (e) $30.0 \mathrm{e} / \mathrm{nm}^{2} \cdot \mathrm{s}$. The DSPC monolayer was formed on a collodion-coated grid for transmission electron microscopic observation (TEM-grid). The selected area was $7.9 \mu \mathrm{m}^{2}$, corresponding to $1 \mu \mathrm{m}$ in diameter, and the exposure time was five s. Arrowheads indicate diffraction peaks corresponding to the carbon-chain packing. To improve the visibility, we have shown the black/white-inverse images.

monolayer, which forms an ordered phase at air-water interface ${ }^{39}$. Because the DSPC monolayer provides a clear reflection, it is convenient for optimisation. We set the wavelength and image acquisition time to be $0.0037 \mathrm{~nm}$ (acceleration voltage $V_{\text {acc }}=100 \mathrm{keV}$ ) and five s, respectively. The sample was kept at $-180{ }^{\circ} \mathrm{C}$ with a cooling holder and the beam damage was reduced.

Figure 2 shows the diffraction patterns from a DSPC monolayer at different electron fluxes. The irradiation at $30.0 \mathrm{e} / \mathrm{nm}^{2} \cdot \mathrm{s}$ gave rise to a broad ring pattern (indicated by arrowheads in Fig. 2e), indicating immediate disruption of the chain-packing structure upon such strong irradiation. Some strong spots appearing in the wide angular regions are unlikely to have originated from the carbon chains because their peak positions $\left(>3.0 \mathrm{~nm}^{-1}\right.$; Supplementary Fig. S1) are far from the lattice spacing of the chain packing. Next, we reduced the electron flux to less than $15.9 \mathrm{e} / \mathrm{nm}^{2} \cdot \mathrm{s}$ and successfully obtained a sharp Debye-Scherrer diffraction at $2.40 \pm 0.01 \mathrm{~nm}^{-1}$ (indicated by arrowheads in Fig. $2 \mathrm{a}-\mathrm{d}$ and their one-dimensional profiles are shown in the bottom of Fig. 3a-d). By applying the lattice spacing $d=0.42 \mathrm{~nm}(=1 / 2.40 \mathrm{~nm})$ to Eq. (2) that is described in "Materials and methods", we calculated the lateral molecular area of the DSPC to be $0.41 \mathrm{~nm}^{2}$. This value is close to the molecular area of the DSPC on the water subphase obtained in the $\pi-A$ isotherm measurement $\left(0.45 \mathrm{~nm}^{2}\right.$ at $\pi=30 \mathrm{mN} / \mathrm{m}$; a black isotherm in Fig. 4a). Also, this result is likely reasonable because it was previously reported that that the chainpacking structure of an ordered membrane (the gel phase) is hardly affected by dehydration ${ }^{25}$.

Figure $3 \mathrm{a}-\mathrm{d}$ shows one-dimensional ED profiles upon continuous irradiation with electron beams, demonstrating that the peak intensity decreases as the irradiation duration $t_{\mathrm{irr}}$ increases. To quantitatively analyse the structural decay, we plotted the normalised intensity $I_{\text {norm }}$ as a function of $t_{\text {irr }}$ (Fig. 3e). Fitting these data to a linear function (dashed lines in Fig. 3e) revealed that the peak intensity decreases in the initial five seconds by $0.4 \pm 5 \%, 2.8 \pm 2 \%, 5.8 \pm 4 \%$, and $15.0 \pm 7 \%$ at electron fluxes of $0.9 \mathrm{e} / \mathrm{nm}^{2} \cdot \mathrm{s}, 2.3 \mathrm{e} / \mathrm{nm}^{2} \cdot \mathrm{s}, 4.7 \mathrm{e} / \mathrm{nm}^{2} \cdot \mathrm{s}$, and $15.9 \mathrm{e} /$ $\mathrm{nm}^{2} \cdot \mathrm{s}$, respectively. Considering their errors, we concluded that the structural decay is sufficiently small to be considered negligible upon irradiation with an electron flux of less than $4.7 \mathrm{e} / \mathrm{nm}^{2} \cdot \mathrm{s}$.

Moreover, we examined the effect of the wavelength $\lambda_{\mathrm{e}}$ on the structural decay by comparing the red plots in Fig. $3 \mathrm{e}\left(\lambda_{\mathrm{e}}=0.0037 \mathrm{~nm} ; V_{\mathrm{acc}}=100 \mathrm{keV}\right)$ and in Supplementary Fig. S2 $\left(\lambda_{\mathrm{e}}=0.0021 \mathrm{~nm} ; V_{\mathrm{acc}}=200 \mathrm{keV}\right)$. Consequently, the decrease in the $I_{\text {norm}}$-value became moderate at the shorter wavelength. However, such shorter wavelength caused a large deviation in the time curve of the $I_{\text {norm }}$-value (Supplementary Fig. S2). Probably, our TEM could not supply stable electron beam under the high acceleration voltage $\left(V_{\text {acc }}=200 \mathrm{keV}\right)$ and, thus, the structural decay of the sample did not show a linear correlation with $t_{\text {irr }}$. Taking account of these results, we used a beam flux of less than $4.7 \mathrm{e} / \mathrm{nm}^{2} \cdot \mathrm{s}$ and a wavelength of $0.0037 \mathrm{~nm}\left(V_{\mathrm{acc}}=100 \mathrm{keV}\right)$ in the following ED experiments.

Macroscopic phase separation in DSPC/DOPC binary monolayers. While there are some previous studies using DSPC/DOPC binary monolayers ${ }^{39,40}$, their phase behaviour remains unknown. Here, we investigated the composition-dependent phase behaviour of the DSPC/DOPC monolayers by surface pressure-molecu- 
a

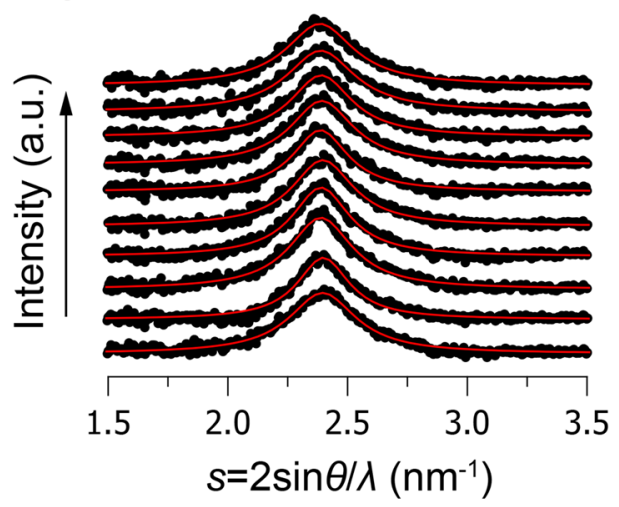

C

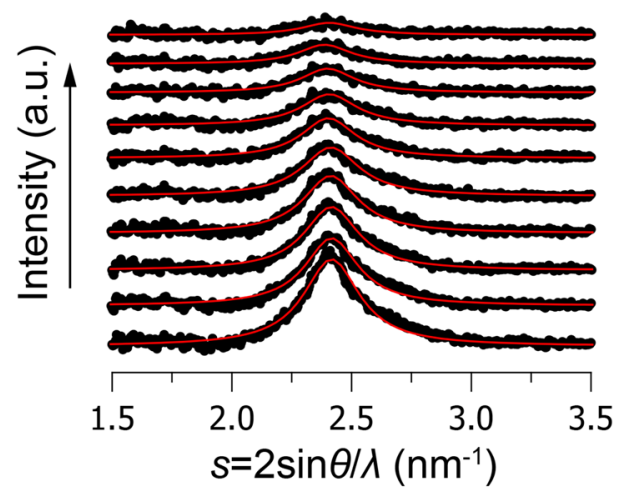

e

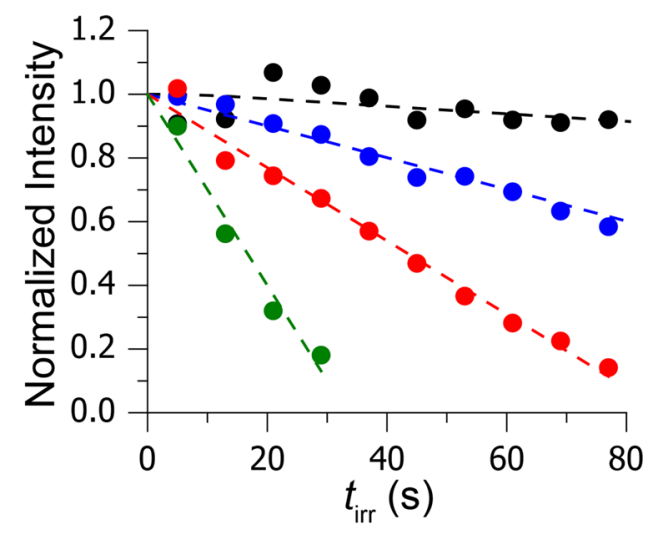

b

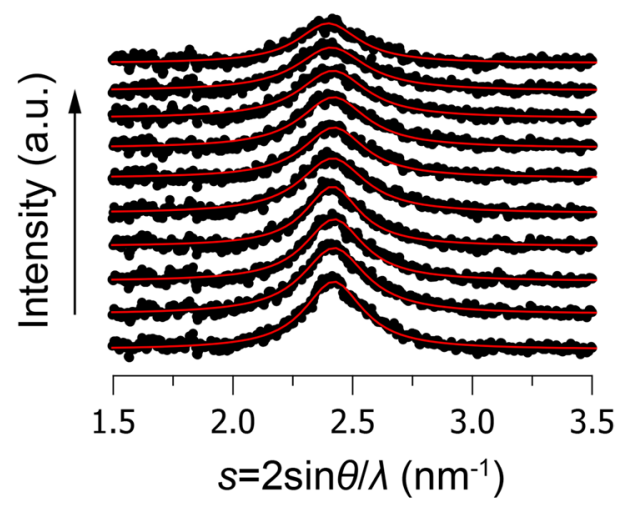

d

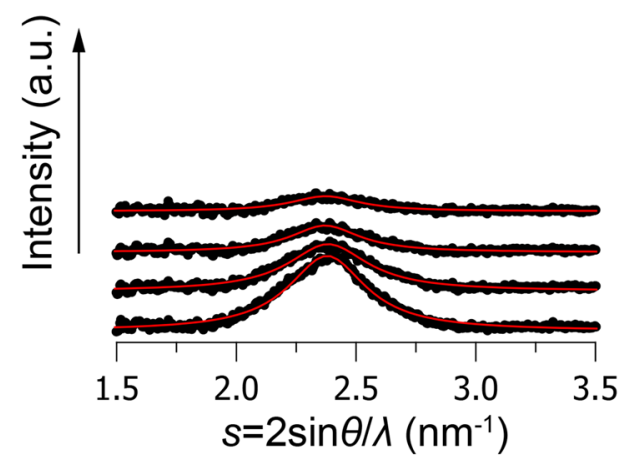

Figure 3. Kinetics of structural decay upon continuous irradiation with an electron beam. One-dimensional ED patterns at the electron flux of (a) $0.9 \mathrm{e} / \mathrm{nm}^{2} \cdot \mathrm{s}$, (b) $2.3 \mathrm{e} / \mathrm{nm}^{2} \cdot \mathrm{s}$, (c) $4.7 \mathrm{e} / \mathrm{nm}^{2} \cdot \mathrm{s}$, and (d) $15.9 \mathrm{e} / \mathrm{nm}^{2} \cdot \mathrm{s}$ are shown. The data were obtained in the same region under continuous irradiation with the electron beam. The irradiation times $t_{\text {irr }}$ were $5 \mathrm{~s}, 13 \mathrm{~s}, 21 \mathrm{~s}, 29 \mathrm{~s}, 37 \mathrm{~s}, 45 \mathrm{~s}, 53 \mathrm{~s}, 61 \mathrm{~s}, 69 \mathrm{~s}$, and $77 \mathrm{~s}$ from the bottom to top profiles. Each profile is fitted to a Lorentz function, and the fitting result is shown by a red profile. The selected area is $0.79 \mu \mathrm{m}^{2}$, which corresponds to $1 \mu \mathrm{m}$ in diameter. (e) Peak heights are plotted as a function of $t_{\text {irr }}$ under the electron flux of $0.9 \mathrm{e} / \mathrm{nm}^{2} \cdot \mathrm{s}$ (black), $2.3 \mathrm{e} / \mathrm{nm}^{2} \cdot \mathrm{s}$ (blue), $4.7 \mathrm{e} / \mathrm{nm}^{2} \cdot \mathrm{s}$ (red), and $15.9 \mathrm{e} / \mathrm{nm}^{2} \cdot \mathrm{s}$ (green). The dashed lines indicate linear fitting in the region $t_{\text {irr }}<77 \mathrm{~s}$ for $0.9 \mathrm{e} / \mathrm{nm}^{2} \cdot \mathrm{s}-4.7 \mathrm{e} / \mathrm{nm}^{2} \cdot \mathrm{s}$ and $t_{\text {irr }}<29 \mathrm{~s}$ for $15.9 \mathrm{e} / \mathrm{nm}^{2} \cdot \mathrm{s}$.

lar area isotherm ( $\pi-A$ isotherm) measurements. Figure $4 \mathrm{a}$ shows the $\pi-A$ isotherms of monolayers consisting of DSPC, DOPC, and their mixtures. The surface pressure of the pure DSPC and pure DOPC monolayers increased steadily as the monolayers were laterally compressed (black and pink isotherms, respectively, in Fig. 4a). On the other hand, DSPC/DOPC monolayers $\left(0.2 \leq x_{\mathrm{DSPC}} \leq 0.7\right)$ show a stepwise increase in surface pressure. This result indicates that the DSPC/DOPC monolayers undergo macroscopic phase separation; namely, the DOPC-rich disordered domains collapse at lower surface pressure (indicated by an arrow in Fig. 4a) and the DSPC-rich 
a

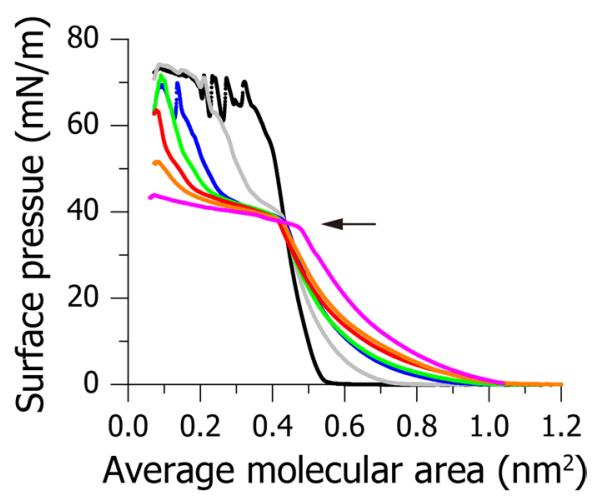

b

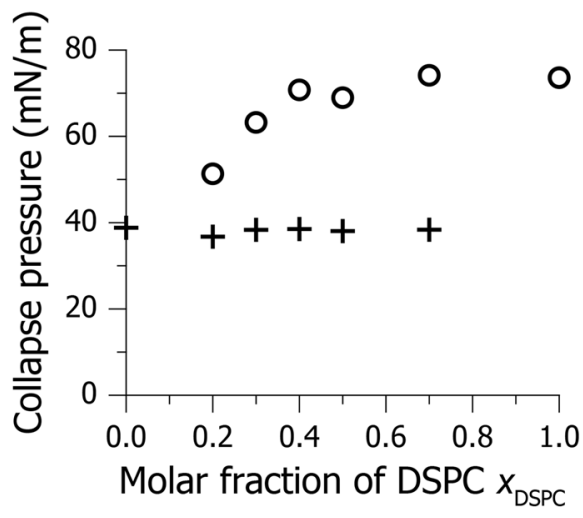

Figure 4. Composition-dependent phase behaviour of DSPC/DOPC binary monolayers. (a) $\pi-A$ isotherms of DSPC/DOPC binary monolayers at different mole fractions of DSPC $\left(x_{\mathrm{DSPC}}\right) \cdot x_{\mathrm{DSPC}}=0$ (pink), 0.2 (orange), 0.3 (red), 0.4 (green), 0.5 (blue), 0.7 (grey), and 1.0 (black). An arrow indicated the collapse pressure of the disordered domains. (b) Collapse pressure vs. composition plot. The circles and crosses show the collapse pressure of the ordered and disordered domains, respectively.

ordered domains at higher surface pressure $(\pi>40 \mathrm{mN} / \mathrm{m})^{5}$. Here, the disordered domain is formed by almost pure DOPC because the collapse pressure of the disordered domain $(\pi \sim 40 \mathrm{mN} / \mathrm{m})$ is consistent with that of the pure DOPC monolayer across the experimental compositional range (crosses in Fig. 4b). On the other hand, in the region of $0.2 \leq x_{\mathrm{DSPC}}<0.4$, the collapse pressure of the ordered domain is significantly smaller than that of pure DSPC (circles in Fig. 4b), indicating that the ordered domain does not consist of pure DSPC, but contains small amounts of DOPC. In the following experiments, we analyse the lipid packing structure of the ordered domain formed in the DSPC/DOPC $\left(x_{\mathrm{DSPC}}=0.3\right)$ monolayer because the structure of the pure DSPC monolayer has already been obtained in Figs. 2 and 3.

Chain packing structures inside a single ordered membrane domain. Figure 5 a shows a fluorescent micrograph of the DSPC/DOPC monolayer, which is formed on the collodion-coated TEM grid. Since this sample contains $0.2 \mathrm{~mol} \%$ Texas Red-DPPE, a disordered phase marker ${ }^{41}$, the darker and brighter regions correspond to the DSPC-rich ordered and DOPC-rich disordered domains, respectively. In the following experiments, we determined the beam positions, referring to the domain distribution shown in the fluorescent micrographs (see "Materials and methods" and Supplementary Fig. S3 for details).

Figure $5 \mathrm{~b}$ shows the ED patterns from the $0.79 \mu \mathrm{m}^{2}$ region inside the ordered domain, giving rise to sharp hexagonal spots. To gain further insight into the chain-packing structure, we represented the one-dimensional intensity of the hexagonal spots and found that the ordered domain gives a single sharp peak at $2.40 \mathrm{~nm}^{-1}$ (Fig. 5d). These results revealed that the carbon chains aligned perpendicular to the monolayer surface with a lattice spacing of $0.42(=1 / 2.40) \mathrm{nm}$. On the other hand, the disordered domain, which consists of almost pure DOPC, gave a broad Debye-Scherrer pattern centred at $2.26 \mathrm{~nm}^{-1}$ (Fig. $5 \mathrm{c}$ and a red profile in Fig. 5e). This ED pattern is similar to the WAXD pattern of the disordered DOPC bilayers ${ }^{42}$; DOPC bilayers also give a broad WAXD peak and the peak position is roughly estimated to be $2.23 \mathrm{~nm}^{-1}$. This fact indicates that the RFS protocol does not cause significant shrinkage of the chain-packing lattice in the disordered monolayer, being in line with FRET experiments (Fig. 1). 

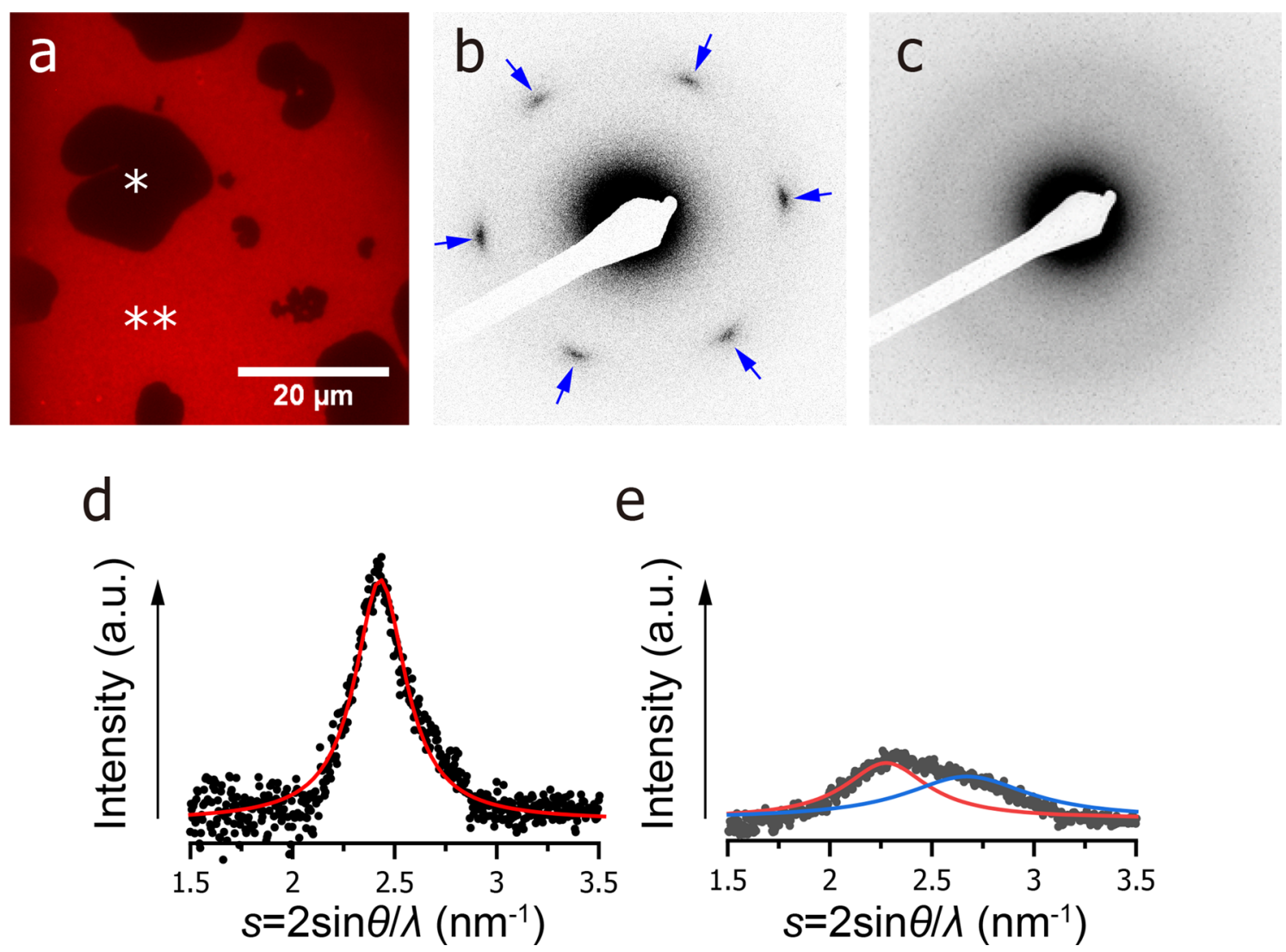

Figure 5. Selective acquisition of ED patterns from the ordered and disordered domains. (a) A fluorescent micrograph of the DSPC/DOPC $\left(x_{\mathrm{DSPC}}=0.3\right)$ monolayer formed on a collodion-coated TEM-grid. Since sample contain $0.2 \mathrm{~mol} \%$ Texas Red-DPPE, a disordered domain marker, the $\left(^{*}\right)$ darker and $\left({ }^{*}\right)$ brighter regions correspond to the ordered and disordered domains, respectively. (b) and (c) show ED patterns obtained in the ordered and disordered domains, respectively. Arrows indicate the hexagonal spot obtained in the ordered domain. The selected area is $0.79 \mu \mathrm{m}^{2}$, corresponding to a diameter of $1 \mu \mathrm{m}$. An exposure time of five seconds was used. (d) and (e) show the one-dimensional ED profiles of (b) and (c), respectively. Although we subtracted the background using a monolayer-free TEM-grid (see "Materials and methods" for details), the diffraction peak from the collodion film could not be completely removed in the panel (e). This is because the thickness of the collodion film is not homogeneous on the TEM-grid. Thus, we deconvoluted those peaks using the two Lorentz functions; the red and blue profiles show the diffraction peaks corresponding to the carbon chains and the collodion film, respectively.

Finally, taking advantage of the small size of the selected areas in the ED experiments, we compared the chain packing structure at seven different regions across a single ordered domain by low-flux scanning electron diffraction (LFSED). In the LFSED experiment, we reduced the electron flux and exposure time to be 0.8 e/ $\mathrm{nm}^{2} \cdot \mathrm{s}$ and $0.5 \mathrm{~s}$, respectively, for further suppression of the beam damage. Then, the size of each region was set to $6.2 \mu \mathrm{m}^{2}$. As in Fig. 5b, sharp hexagonal spots were obtained in the ordered domain (regions 3-6 in Fig. 6b,c), while a broad Debye-Scherrer pattern was observed in the disordered domain (regions 1, 2, and 7 in Fig. 6b,c). Moreover, the one-dimensional ED pattern showed that the peak positions $\left(2.39 \pm 0.02 \mathrm{~nm}^{-1}\right)$ are almost the same between regions 3-6 (Supplementary Fig. S4), indicating a similar lattice spacing of the chain packing in the ordered domain. To analyse substructures formed in the ordered domains, we plotted the peak intensity at $2.39 \mathrm{~nm}^{-1}$ along the azimuthal direction $\theta$. Consequently, it was found that the centre regions of the ordered domain (regions 4 and 5) show diffraction peaks at almost the same angles (dashed line in Fig. 6d). On the other hand, the regions 3 and 6 showed diffraction peaks at slightly different angles from the centre regions: $+12^{\circ}$ and $-14^{\circ}$, respectively, (arrowheads in Fig. $6 \mathrm{~d}$ ). These results suggest that a single ordered domain contains multiple subdomains with different crystallographic axes. Here, the regions 4 and 5 belong likely to a same subdomain because it is quite unlikely that two different subdomains are incidentally oriented in the same direction. In such a case, the size of the subdomain is larger at the domain centre than in the vicinity of the phase boundary (Fig. 7).

\section{Discussion}

In the present study, we examined the packing structure of lipid carbon chains inside a single ordered domain using the LFSED and identified that the ordered domain consists of multiple subdomains with different crystallographic axes (Fig. 6d). Moreover, results indicated that the size of the subdomains is larger in the domain centre than in the vicinity of the phase boundary (Figs. 6 and 7). Because the edge of the ordered domain is exposed to 


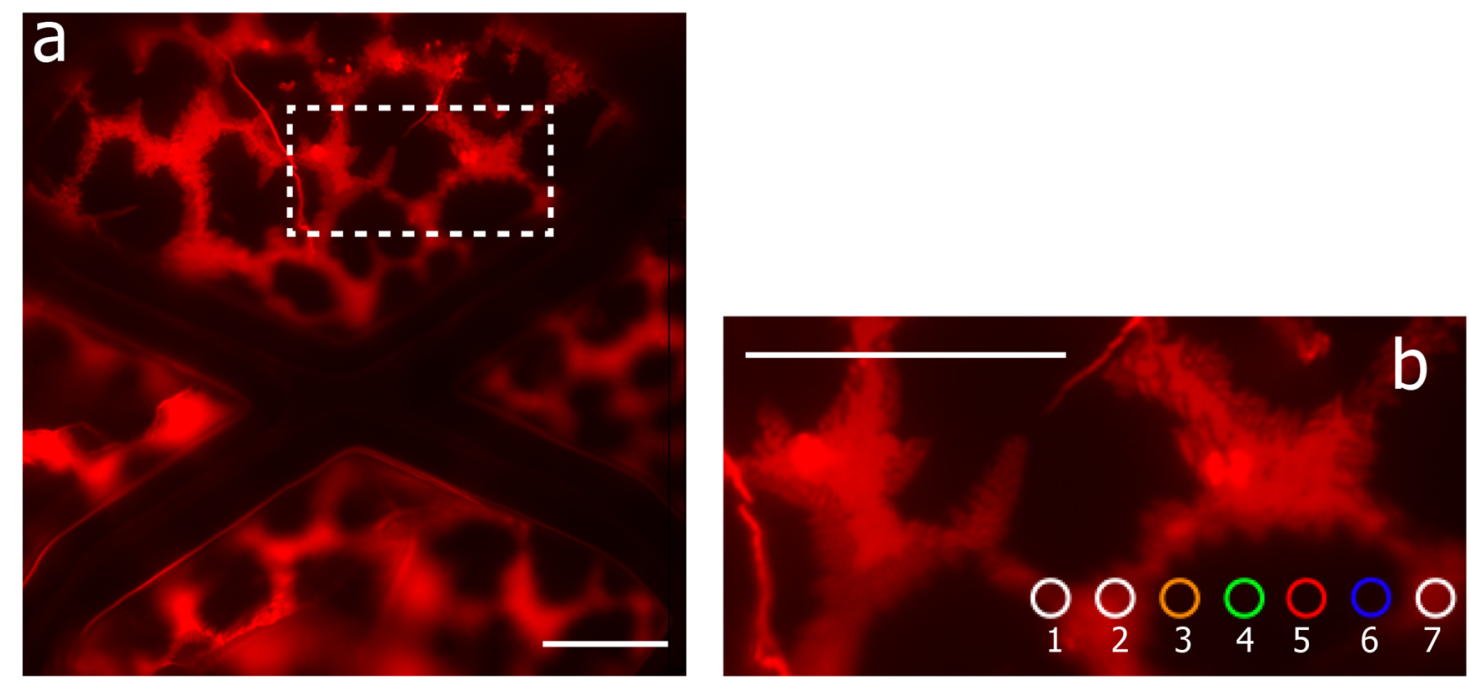

C
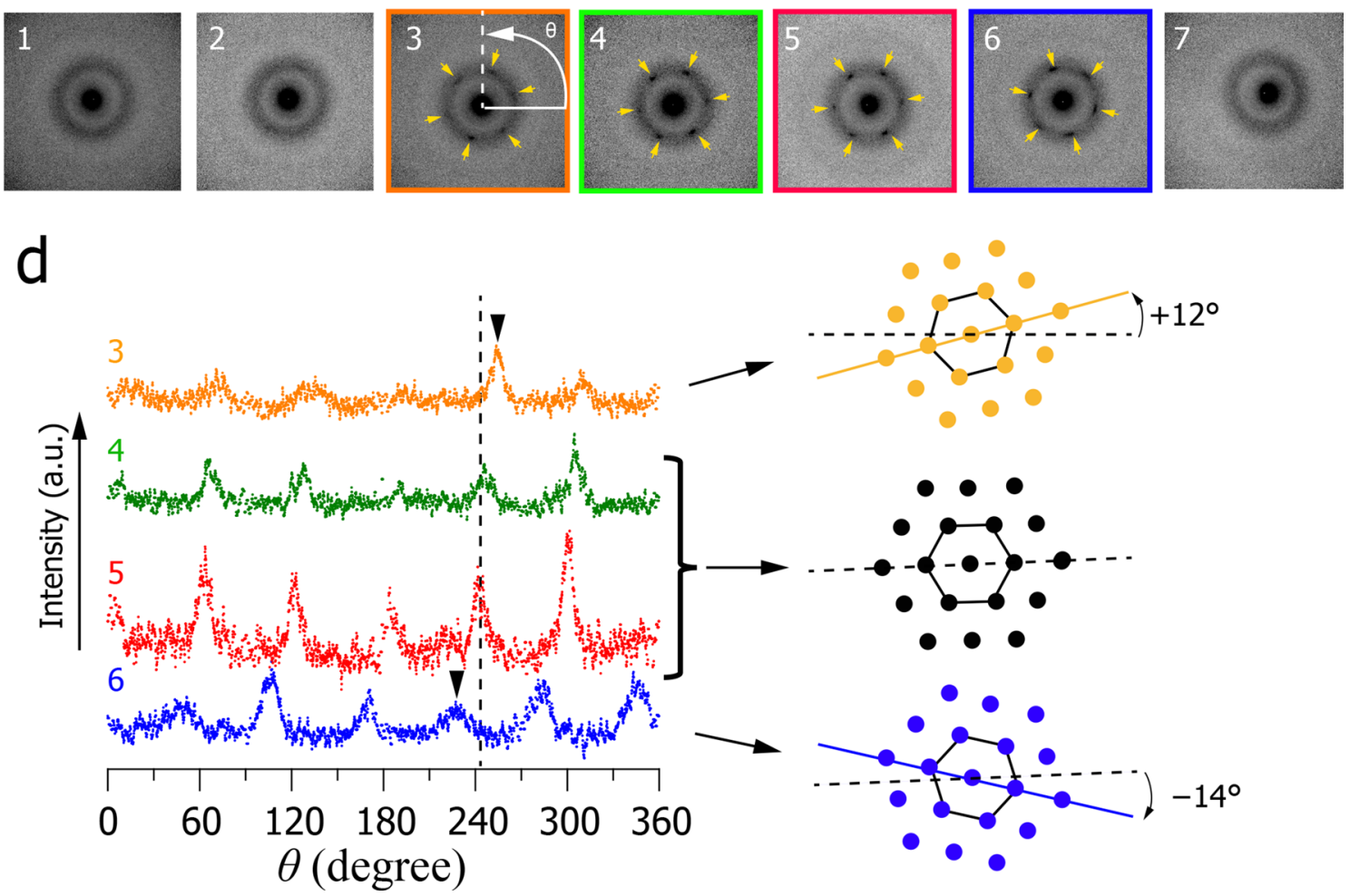

Figure 6. Local structure inside an ordered domain. (a) A fluorescent micrograph of the DSPC/DOPC $\left(x_{\mathrm{DSPC}}=0.3\right)$ monolayer formed on a collodion-coated TEM grid. The darker and brighter regions correspond to the DSPC-rich ordered and DOPC-rich disordered domains, respectively. (b) Magnification of the area, which is indicated by the dashed square in (a). Bars indicate $30 \mu \mathrm{m}$. (c) The ED patterns obtained at regions $1-7$, as indicated in (b). The selected area is $6.2 \mu \mathrm{m}^{2}$, corresponding to $2.8 \mu \mathrm{m}$ in diameter. The corresponding region numbers were directly indicated in the panels. (d) We plotted the intensity of the hexagonal spots appearing along the azimuthal direction $\theta$, which is shown in panel (c). The corresponding region numbers are directly indicated in the panel. The difference in the azimuthal angle of the hexagonal spots between the centre (regions 4 and 5) and annular (regions 3 and 6) regions in the ordered domain are indicated by a dashed line and arrowheads, respectively. Schematic illustrations of the directions of the chain packing lattice in regions 3-6 are also shown in (d).

a disordered matrix, the packing structure near the phase boundary should be directly affected by inter-domain interactions, such as electrostatic interaction ${ }^{43}$, line tension ${ }^{44}$, and transverse shear when the domains are in motion. It is probable that these interactions hamper the unidirectional arrangement of the chain packing, giving rise to the formation of small subdomains in the vicinity of the phase boundary. 


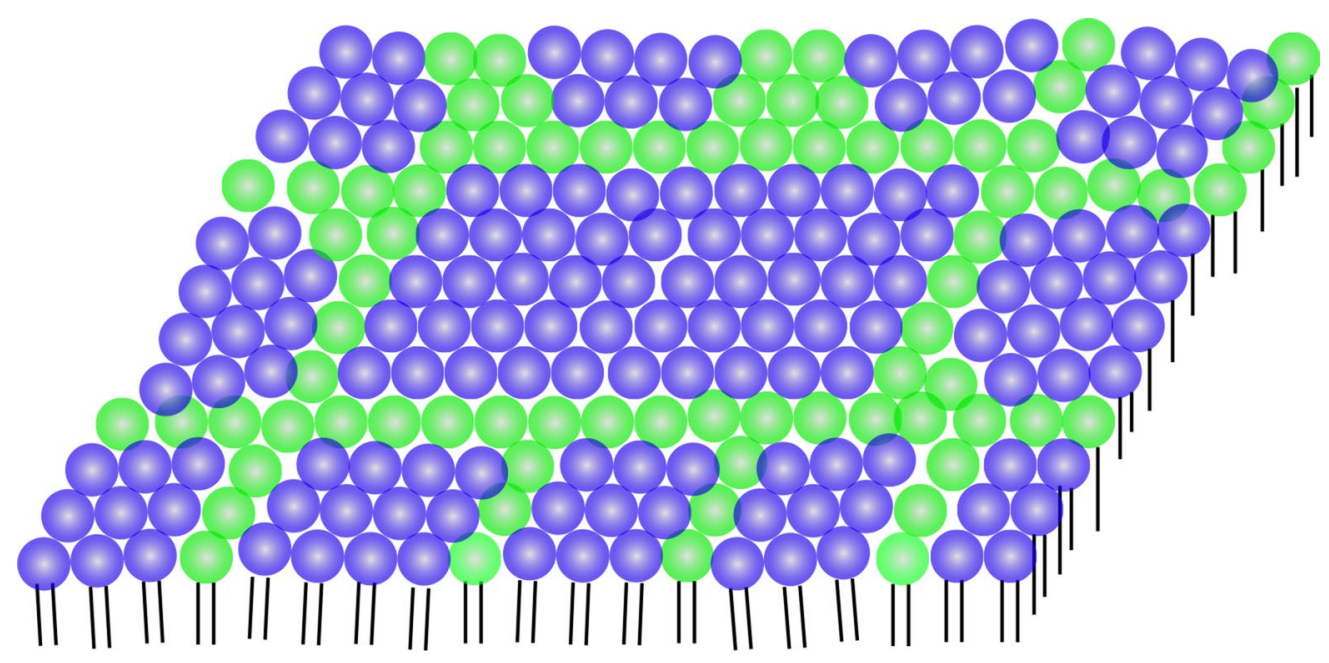

Figure 7. A schematic illustration of the distribution of the subdomains inside a single ordered domain. Blue and green lipids correspond to DSPC and DOPC, respectively.

Some previous studies compared the size of the subdomains based on the halfwidth of the WAXD peak ${ }^{45,46}$. However, these studies also suggested that the width of the WAXD peak depends not only on the size of subdomains but also on the other factors such as the order of the chain packing, the instrumental setup, and so on. In that case, the deconvolution of these components is necessary for estimating the size of the subdomain ${ }^{47}$. On the other hand, the LFSED can assess the size of the subdomain without the deconvolution analysis and, thus, this point is remarkable merit for the LFSED when it is compared with WAXD.

Another interesting finding is that the lattice spacing of the carbon chains is almost the same between the pure DSPC monolayer $\left(2.40 \pm 0.01 \mathrm{~nm}^{-1}\right.$; bottom profiles in Fig. $\left.3 \mathrm{a}-\mathrm{d}\right)$ and the DSPC-rich ordered domain $\left(2.39 \pm 0.02 \mathrm{~nm}^{-1}\right.$; Supplementary Fig. S4), although the $\pi-A$ isotherm measurements showed that the DSPC-rich macro-domain contains small amounts of DOPC (see Fig. $4 \mathrm{~b}$ and Text). We speculated that, in the DSPC-rich domain, the subdomains are formed by pure DSPC, while small amounts of DOPC molecules reside in the intersubdomain space (Fig. 7). In this model, since DOPC molecules do not perturb the chain packing of DSPC, the lattice spacing of the DSPC-rich domain should be consistent with that of the pure DSPC monolayer.

Moreover, the pure DSPC monolayer showed a Debye-Scherrer pattern, while the DSPC-rich ordered domain gave the hexagonal spots (compare Figs. 2 and $5 b$ ). This means that the size of the subdomain is larger in the DSPC-rich domain than in the pure DSPC monolayer. It is not surprising that even in the pure monolayer, incidentally-created membrane defects often chop off the long-range alignment of carbon chains ${ }^{48}$, leading to the formation of subdomains. Here, the question arises as to why the size of the subdomains is different between the pure DSPC monolayer and the DSPC-rich ordered domain. In the pure system, the van der Waals interaction between the DSPC molecules could be the dominant force for DSPC assembly. On the other hand, in the DSPC-rich domain, molecular contact of the unfavourable pair, DSPC and DOPC, should cause energetic disadvantage ${ }^{49}$. We speculate that, besides the van der Waals interaction, avoidance of unfavourable pairing leads to the formation of a larger subdomain in the DSPC-rich domains. However, to confirm this speculation, we need further study on the correlation between the inter-lipid interactions and the size distribution of the subdomains.

It has been suggested that the substructures inside ordered membrane domains are involved in important biological functions. For example, Wu et al. demonstrated that molecular dynamics in the ordered domains are strongly affected by the substructure and, thus, proposed that the subdomains are responsible for determining the protein partitioning into the ordered domains ${ }^{17}$. In addition, our group reported that a minor structural change of a lipid molecule alters the size of the subdomains, affecting water permeability of the membranes ${ }^{46}$. However, these previous studies could not address the chain packing structure of each subdomain nor the determination of the subdomain size. Therefore, LFSED is promising methodology to understand mechanistic link between the substructure of the ordered domains and their biological functions.

\section{Conclusion}

Electron diffraction (ED) is promising methodology for examining local structures of the lipid membranes due to following reasons. Firstly, ED can assess local structures of lipid membranes. Secondly, due to great diffraction potency of the electron beam, ED can produce strong diffraction peaks even from a single membrane domain. Lastly, ED is easily performed with a usual transmission electron microscope. Compared with WAXD, these points are striking advantages of ED. However, some technical difficulties hamper application of the ED to lipid membranes. For example, the ED experiment requires a dry and fixed sample. In addition, the electron beam causes significant damage to the lipid membranes.

In the present study, we first prepared dehydrated samples using the RFS method and demonstrated that it provides dehydrated monolayer samples without significant shrinkage of the chain-packing lattice in the monolayer samples. We next optimised the intensity of the electron beam to minimise the beam damage to the membrane sample. As a result, we found that the beam damage is sufficiently small to be considered negligible 
under the electron flux and irradiation durations of less than $4.7 \mathrm{e} / \mathrm{nm}^{2} \cdot \mathrm{s}$ and $5 \mathrm{~s}$, respectively. Finally, we examined the chain packing structure inside the ordered domain, which is formed in the DSPC/DOPC $\left(x_{\mathrm{DSPC}}=0.3\right)$ phase separated monolayer. Consequently, our LFSED measurement indicated that the ordered domain consists of multiple subdomains with different crystallographic axes (Fig. 6d). Moreover, it is indicated that the size of the subdomain is larger in the domain centre than in the vicinity of the phase boundary (Fig. 7). To the best of our knowledge, this is the first example to address the chain packing structures inside a single ordered domain.

\section{Materials and methods}

Materials. 1, 2-distearoyl-sn-3-phosphatidylcholine (DSPC) and 1, 2-dioleoyl-sn-3-phosphatidylcholine (DOPC) were purchased form Avanti Polar Lipid Inc. (Alabaster, AL). Fluorescent lipid analogues, 2-(4,4-difluoro-5,7-dimethyl-4-bora-3a,4a-diaza-s-indacene-3-pentanoyl)-1-hexadecanoyl-sn-glyceo-3-phosphocholine (Bodipy-PC) and N-(Texas Red sulfonyl)-1,2-dihexadecanoyl-snglycero-3-phosphatidylethanolamine triethylammonium salt (Texas Red-DPPE) were purchased from Molecular Probe (Eugene, OR). These lipids and fluorescent lipid analogues were dissolved in chloroform/methanol (4:1 v/v) (Wako pure chemicals, Osaka, Japan) and stored at $-40^{\circ} \mathrm{C}$ until use.

Surface pressure vs. molecular area isotherm ( $\pi-A$ isotherm) measurements and preparation of supported monolayers. Monolayers of lipid mixtures were prepared on a computer-controlled Langmuir film balance (USI System, Fukuoka, Japan) calibrated using stearic acid (Sigma-Aldrich, St. Louis, MO). The lipid solution was prepared by dissolving appropriate amount of DSPC and DOPC in chloroform/methanol $(4: 1 \mathrm{v} / \mathrm{v})$. Then, $30 \mu \mathrm{L}$ of lipid solution $(1 \mathrm{mg} / \mathrm{mL})$ was spread onto the water subphase $\left(100 \times 290 \mathrm{~mm}^{2}\right)$ with a glass micropipette (Drummond Scientific Company, Pennsylvania, USA). The monolayers were compressed at a rate of $20 \mathrm{~mm}^{2} / \mathrm{s}$ after an initial delay period of $10 \mathrm{~min}$ for the evaporation of organic solvents. The subphase and ambient temperatures were controlled at $25.0 \pm 0.1^{\circ} \mathrm{C}$ and $25 \pm 1{ }^{\circ} \mathrm{C}$, respectively. The measurements were repeated three times under the same conditions and average data were shown.

In the preparation of the supported monolayer, the lipid solution in the presence of $0.2 \mathrm{~mol} \%$ Texas Red-DPPE was spread on the water subphase. Then, the substrate such as a mica plate and a collodion coated TEM-grid (Okenshoji Co., Ltd, Tokyo, Japan) was horizontally dipped into the water subphase, and the lipid sample was compressed to $30 \mathrm{mN} / \mathrm{m}$ at the rate of $20 \mathrm{~mm}^{2} / \mathrm{s}$. After the compression, the monolayer was transferred onto the substrate. Then, distribution of the ordered and disordered phases in the supported monolayers were observed with a fluorescent microscope BZ-X700 (Keyence, Osaka, Japan).

Rapid freeze and sublimation (RFS) methods. The dehydrated sample for the electron diffraction experiment was prepared by the RFS method, which is derived from the methods employed in freeze-fracture electron microscopy and mass-based imaging ${ }^{36-38}$. Briefly, immediately after the preparation of the supported monolayer, the wet sample was rapidly frozen in slush $\mathrm{N}_{2}$. Then, the sample was stored in a vacuum chamber maintained at $6.7 \times 10^{-2} \mathrm{~Pa}$, which is much lower than the triple point of water in the pressure vs. temperature phase diagram. The temperature was gradually increased from -180 to $25{ }^{\circ} \mathrm{C}$ to completely sublimate water molecules from the sample.

Fluorescent energy transfer (FRET) experiments. Mica supported DOPC monolayers were prepared by aforementioned protocol. In the FRET measurements, the samples contained 0.2 mol\% Bodipy-PC and $0.4 \mathrm{~mol} \%$ Texas Red-DPPE as FRET donor and acceptor, respectively. Then, the samples were dehydrated by the RFS methods or dehydrated at the atmospheric pressure and the room temperature. Then, the intensity of the FRET-donor $I_{\text {donor }}$ was measured at more than thirty different locations $\left(50 \times 50 \mu \mathrm{m}^{2}\right.$ for each $)$ in the sample with fluorescent microscope BZ-X700 and the average value was shown. The $I_{\text {donor-value of the wet sample was }}$ measured immediately after the preparation of the supported monolayer. The excitation wavelength of $470 \mathrm{~nm}$ was applied to Bodipy-PC and the emission were detected at $525 \mathrm{~nm}$ using dichroic mirrors OP-87763 (Keyence, Osaka, Japan).

Electron diffraction (ED) experiments. The local-structure analysis inside a single membrane domain was conducted with transmission electron microscope JEM1400 (JEOL, Tokyo, Japan), and other ED experiments were conducted with JEM2100HCKM (JEOL Ltd., Tokyo, Japan). The supported monolayer mounted on a cooling folder, such as Gatan 626 and Gatan 636 for JEM1400 and JEM2100HCKM, respectively, was set into an electron microscope. The sample was kept at $-180^{\circ} \mathrm{C}$, and the electron beam was irradiated perpendicular to the supported monolayer surface. The instrumental camera length was calibrated using gold particles. Electron diffraction experiments were performed at an accelerating voltage of $100 \mathrm{kV}$ unless otherwise mentioned. The other conditions such as electron flux and exposure time were described in the text. Diffraction patterns were acquired using a CCD camera ES500W (Gatan Inc., Pleasanton, CA) and TemCam-F416 (TVIPS, Gauting, Germany) for JEM1400 and JEM2100HCKM, respectively. To improve visibility of the diffraction patterns, we showed black-and-white inverse images. The value of the electron flux was estimated from the brightness of the digitised image of the incident beam in the absence of a lipid sample (blank image) because the electron flux was too low to be detected by the equipped current meter ${ }^{35}$. Specifically, in the LFSED measurements, the electron flux was calculated by interpolating the brightness of the blank image at $0 \mathrm{pA}$ and $1 \mathrm{pA}$. Before all experiments, we took a warming-up duration for more than two hours to supply stable electron flux. Referring to the fluorescent micrograph, we applied an electron beam to the target together with acquisition of the diffraction pattern. After the experiment, we determined the beam position using the debris and incidental breaks on the collodion film as landmarks (Supplementary Fig. S3). The diffraction patterns were linearised using Fit 2D and 
one-dimensional profiles as a function of $s=1 / \mathrm{d}=2 \sin \theta / \lambda$ were obtained. Here, $s$ is modulus of scattering vector, $d$ is real spacing of the packing lattice, $\theta$ is the scattering angle and $\lambda$ is the wavelength of the electron beam. The diffraction peak of the collodion film was subtracted from all the data using a blank sample, which is monolayerfree TEM-grid. The peak position and intensity were estimated by fitting the diffraction profile to the Lorentz function with Origin 2015 (Lightstone, Tokyo, Japan).

According to previous literature ${ }^{24}$, the lateral occupied area of a lipid molecule $\left(A_{\text {lipid }}\right)$ is estimated by

$$
A_{\text {lipid }}=\frac{2 \times A_{\text {chain }}}{\cos \theta} .
$$

Here, $A_{\text {chain }}$ is the lateral occupied area of a carbon chain, and $\theta$ is the tilt angle of the carbon chain to the membrane normal. If the tilt angle of carbon chains is almost zero, the lateral occupied area of the lipid is easily estimated by

$$
A_{\text {lipid }}=\frac{4}{\sqrt{3}} d^{2}
$$

Received: 12 June 2020; Accepted: 23 November 2020

Published online: 21 December 2020

\section{References}

1. Lingwood, D. \& Simons, K. Lipid rafts as a membrane-organizing principle. Science 327, 46-50 (2010).

2. Suzuki, K. G. N. et al. Transient GPI-anchored protein homodimers are units for raft organization and function. Nat. Chem. Biol. 8, 774-783 (2012).

3. Nicolson, G. L. The fluid-mosaic model of membrane structure: Still relevant to understanding the structure, function and dynamics of biological membranes after more than 40 years. Biochim. Biophys. Acta Biomembr. 1838, 1451-1466 (2014).

4. Kusumi, A. et al. Defining raft domains in the plasma membrane. Traffic 21, 106-137 (2020).

5. Kinoshita, M., Matsumori, N. \& Murata, M. Coexistence of two liquid crystalline phases in dihydrosphingomyelin and dioleoylphosphatidylcholine binary mixtures. Biochim. Biophys. Acta Biomembr. 1838, 1372-1381 (2014).

6. Goñi, F. M. et al. Phase diagrams of lipid mixtures relevant to the study of membrane rafts. Biochim. Biophys. Acta Mol. Cell Biol. Lipids 1781, 665-684 (2008).

7. Kinoshita, M. et al. Raft-based sphingomyelin interactions revealed by new fluorescent sphingomyelin analogs. J. Cell Biol. 216, 1183-1204 (2017).

8. Klymchenko, A. S. \& Kreder, R. Fluorescent probes for lipid rafts: from model membranes to living cells. Chem. Biol. 21, 97-113 (2014).

9. Kahya, N., Scherfeld, D., Bacia, K., Poolman, B. \& Schwille, P. Probing lipid mobility of raft-exhibiting model membranes by fluorescence correlation spectroscopy. J. Biol. Chem. 278, 28109-28115 (2003).

10. Kinoshita, M. et al. Emphatic visualization of sphingomyelin-rich domains by inter-lipid FRET imaging using fluorescent sphingomyelins. Sci. Rep. 7, 16801 (2017).

11. Veatch, S. L. \& Keller, S. L. Miscibility phase diagrams of giant vesicles containing sphingomyelin. Phys. Rev. Lett. 94, 3-6 (2005).

12. Baumgart, T., Das, S., Webb, W. W. \& Jenkins, J. T. Membrane elasticity in giant vesicles with fluid phase coexistence. Biophys. J. 89, 1067-1080 (2005).

13. Chiantia, S., Kahya, N., Ries, J. \& Schwille, P. Effects of ceramide on liquid-ordered domains investigated by simultaneous AFM and FCS. Biophys. J. 90, 4500-4508 (2006).

14. Ando, J. et al. Sphingomyelin distribution in lipid rafts of artificial monolayer membranes visualized by Raman microscopy. Proc. Natl. Acad. Sci. U.S.A. 112, 4558-4563 (2015).

15. Feigenson, G. W. Phase diagrams and lipid domains in multicomponent lipid bilayer mixtures. Biochim. Biophys. Acta Biomembr. 1788, 47-52 (2009).

16. Veatch, S. L. \& Keller, S. L. Separation of liquid phases in giant vesicles of ternary mixtures of phospholipids and cholesterol. Biophys. J. 85, 3074-3083 (2003).

17. Wu, H. M., Lin, Y. H., Yen, T. C. \& Hsieh, C. L. Nanoscopic substructures of raft-mimetic liquid-ordered membrane domains revealed by high-speed single-particle tracking. Sci. Rep. 6, 20542 (2016).

18. Yasuda, T., Slotte, J. P. \& Murata, M. Nanosized phase segregation of sphingomyelin and dihydrosphigomyelin in unsaturated phosphatidylcholine binary membranes without cholesterol. Langmuir 34, 13426-13437 (2018).

19. Shirota, K. et al. Detection of sphingomyelin clusters by Raman spectroscopy. Biophys. J. 111, 999-1007 (2016).

20. Feigenson, G. W. Pictures of the substructure of liquid-ordered domains. Biophys. J. 109, 854-855 (2015).

21. McIntosh, T. J. Differences in hydrocarbon chain tilt between hydrated phosphatidylethanolamine and phosphatidylcholine bilayers. A molecular packing model. Biophys. J. 29, 237-245 (1980).

22. Tristram-Nagle, S., Liu, Y., Legleiter, J. \& Nagle, J. F. Structure of gel phase DMPC determined by X-ray diffraction. Biophys. J. 83, 3324-3335 (2002).

23. Watanabe, H., Obata, Y., Ishida, K. \& Takayama, K. Effect of l-menthol on the thermotropic behavior of ceramide $2 /$ cholesterol mixtures as a model for the intercellular lipids in stratum corneum. Colloids Surf. B 73, 116-121 (2009).

24. Takahashi, H., Matuoka, S., Kato, S., Ohki, K. \& Hatta, I. Electrostatic interaction of poly(l-lysine) with dipalmitoylphosphatidic acid studied by X-ray diffraction. Biochim. Biophys. Acta 1069, 229-234 (1991).

25. Hui, S. W., Cowden, M., Papahadjopoulos, D. \& Parsons, D. F. Electron diffraction study of hydrated phospholipid single bilayers. Effects of temperature, hydration and surface pressure of the 'precursor' monolayer. Biochim. Biophys. Acta 382, 265-275 (1975).

26. Hui, S. W. \& Yu, H. Electron diffraction studies of molecular ordering and orientation in phospholipid monolayer domains. Biophys. J. 64, 150-156 (1993).

27. Hui, S. W., Parsons, D. F. \& Cowden, M. Electron diffraction of wet phospholipid bilayers. Proc. Natl. Acad. Sci. U.S.A. 71, 5068-5072 (1974).

28. Hui, S. W. \& He, N. B. Molecular organization in cholesterol-lecithin bilayers by X-ray and electron diffraction measurements. Biochemistry 22, 1159-1164 (1983).

29. Fischer, A. \& Sackmann, E. A charge-decoration technique for studying the heterogeneity of coexistent monolayer phases by electron microscopy. Nature 313, 299-301 (1985).

30. Helm, C. A., Möhwald, H., Kjaer, K. \& Als-Nielsen, J. Phospholipid monolayers between fluid and solid states. Biophys. J. 52, 381-390 (1987). 
31. Hui, S. W. Effects of temperature and hydration. Ultramicroscopy 5, 505-512 (1980).

32. Pilgram, G. S. K., Engelsma-van Pelt, A. M., Oostergetel, G. T., Koerten, H. K. \& Bouwstra, J. A. Study on the lipid organization of stratum corneum lipid models by (cryo-) electron diffraction. J. Lipid Res. 39, 1669-1676 (1998).

33. Nakazawa, H. et al. Low-flux electron diffraction study for the intercellular lipid organization on a human corneocyte. Biochim. Biophys. Acta Biomembr. 1828, 1424-1431 (2013).

34. Imai, T., Nakazawa, H. \& Kato, S. Thermal phase transition behavior of lipid layers on a single human corneocyte cell. Chem. Phys. Lipids 174, 24-31 (2013).

35. Nag, K. \& Keough, K. M. Epifluorescence microscopic studies of monolayers containing mixtures of dioleoyl- and dipalmitoylphosphatidylcholines. Biophys. J. 65, 1019-1026 (1993).

36. Shotton, D. M. \& Servers, N. J. 1995.An introduction to freeze fracture and deep etching. in Rapid Freezing, Freeze Fracture, and Deep Etching 1-31 (Wiley, New York, 1995). https://doi.org/10.1016/j.chemphyslip.2009.06.143

37. Marxer, C. G., Kraft, M. L., Weber, P. K., Hutcheon, I. D. \& Boxer, S. G. Supported membrane composition analysis by secondary ion mass spectrometry with high lateral resolution. Biophys. J. 88, 2965-2975 (2005).

38. Kraft, M. L., Weber, P. K., Longo, M. L., Hutcheon, I. D. \& Boxer, S. G. Phase separation of lipid membranes analyzed with highresolution secondary ion mass spectrometry. Sicence 313, 1948-1951 (2006).

39. Hac-Wydro, K., Jedrzejek, K. \& Dynarowicz-Łatka, P. Effect of saturation degree on the interactions between fatty acids and phosphatidylcholines in binary and ternary Langmuir monolayers. Colloids Surf. B 72, 101-111 (2009).

40. del Luzard, M. C., Peltzer, G. \& Disalvo, E. A. Surface potential of lipid interfaces formed by mixtures of phosphatidylcholine of different chain lengths. Langmuir 14, 5858-5861 (1998).

41. Dietrich, C., Volovyk, Z. N., Levi, M., Thompson, N. L. \& Jacobson, K. Partitioning of Thy-1, GM1, and cross-linked phospholipid analogs into lipid rafts reconstituted in supported model membrane monolayers. Proc. Natl. Acad. Sci. U.S.A. 98, 10642-10647 (2001).

42. Smith, E. A., Smith, C., Tanksley, B. \& Dea, P. K. Effects of cis- and trans-unsaturated lipids on an interdigitated membrane. Chem. Phys. 190-191, 1-7 (2014).

43. Huang, J. \& Feigenson, G. W. Monte Carlo simulation of lipid mixtures: finding phase separation. Biophys. J. 65, 1788-1794 (1993).

44. Akimov, S. A., Kuzmin, P. I., Zimmerberg, J. \& Cohen, F. S. Lateral tension increases the line tension between two domains in a lipid bilayer membrane. Phys. Rev. E 75, 011919 (2007).

45. Mills, T. T. et al. Liquid-liquid domains in bilayers detected by wide angle X-ray scattering. Biophys. J. 95, 682-690 (2008).

46. Kinoshita, M., Tanaka, K. \& Matsumori, N. The influence of ceramide and its dihydro analog on the physico-chemical properties of sphingomyelin bilayers. Chem. Phys. Lipid 226, 104835 (2020).

47. de Keijser, T. H., Langford, J. I., Mittemeijer, E. J. \& Vogels, A. B. P. Use of the Voigt function in a single-line method for the analysis of X-ray diffraction line broadening. J. Appl. Crystallogr. 15, 308-315 (1982).

48. Kienle, D. F., De Souza, J. V., Watkins, E. B. \& Kuhl, T. L. Thickness and refractive index of DPPC and DPPE monolayers by multiple-beam interferometry. Anal. Bioanal. Chem. 406, 4725-4733 (2014).

49. Dai, J., Alwarawrah, M., Ali, M. R., Feigenson, G. W. \& Huang, J. Simulation of the lo -ld phase boundary in DSPC/DOPC/cholesterol ternary mixtures using pairwise interactions. J. Phys. Chem. B 115, 1662-1671 (2011).

\title{
Acknowledgements
}

We thank Prof. Satoru Kato (Kwansei-Gakuin University, Hyogo, Japan) for their approval of use of electron microscope for LFSED experiments and useful discussion about analysis of the electron diffraction patterns. The other electron microscopic observations were conducted at Ultramicroscopy Research Center (Kyusyu University, Fukuoka, Japan). This work was supported in part by Grants-in-Aid for scientific research from the Japan Society for the Promotion of Science (Wakate B and Kiban C) to Masanao Kinoshita [17K15107 and 20K06590, respectively]. We would like to thank Editage (http://www.editage.com) for English language editing.

\section{Author contributions}

S.Y. optimised the conditions of the electron diffraction experiments, and S.Y and M.K. performed electron diffraction experiments and surface pressure vs. molecular area isotherm measurements. N.M. conceived and formulated this project and evaluated and discussed data. M.K. and N.M. wrote the manuscript.

\section{Competing interests}

The authors declare no competing interests.

\section{Additional information}

Supplementary Information The online version contains supplementary material available at https://doi. org/10.1038/s41598-020-79083-7.

Correspondence and requests for materials should be addressed to M.K.

Reprints and permissions information is available at www.nature.com/reprints.

Publisher's note Springer Nature remains neutral with regard to jurisdictional claims in published maps and institutional affiliations.

\begin{abstract}
Open Access This article is licensed under a Creative Commons Attribution 4.0 International License, which permits use, sharing, adaptation, distribution and reproduction in any medium or
format, as long as you give appropriate credit to the original author(s) and the source, provide a link to the Creative Commons licence, and indicate if changes were made. The images or other third party material in this article are included in the article's Creative Commons licence, unless indicated otherwise in a credit line to the material. If material is not included in the article's Creative Commons licence and your intended use is not permitted by statutory regulation or exceeds the permitted use, you will need to obtain permission directly from the copyright holder. To view a copy of this licence, visit http://creativecommons.org/licenses/by/4.0/.
\end{abstract}

(C) The Author(s) 2020 